\title{
A Note upon some Properties of the Curve of Striction.
}

By Dr H. W. Richmond, F.R.S., LL.D.

(Received 3rd August 19\%6. Read 5th November 1926.)

Certain properties of Ruled Surfaces relating to their Curves of Striction are suggested and immediately proved by the use of a particular kind of coordinates, known as Dual Coordinates, for the Generating Lines. These coordinates, were introduced by Prof. E. Study; the theory of them is fully explained and many beautiful applications are made in his treatise, Geometrie der Dynamen, Teubner, 1903, and in his article, Complexe Grössen in the Encyclopädie der math. Wissenschaften, Bd. I., pp. 147-183, and specially p. 166 . In applying the method here I have ventured to regard the fundamental quantity $c$ in a light which, if less rigorous, has the advantage of being familiar to most of us in other mathematical work.

1. Dual Coordinates and Dual Numbers. The coordinates of the straight line which passes through a point $(f, g, h)$ and has direction-cosines $(l, m, n)$ are commonly taken to be $(l, m, n, r, q, r)$ where

so that

$$
\begin{aligned}
& p=g n-h m, \\
& q=h l-t m, \\
& r=f m-g l,
\end{aligned}
$$

$$
\begin{aligned}
& l^{3}+m^{2}+n^{2}=1: \\
& l p+m q+n r=0 .
\end{aligned}
$$

Hence, if we agree to write

$$
\begin{aligned}
L & =l+e p \\
M & =m+e q \\
N & =n+e r, \\
L^{2}+M^{2}=N^{2} & =1+e^{2}\left(p^{2}+q^{2}+r^{2}\right) .
\end{aligned}
$$

Now in many applications of mathematics, for example in discussing the oscillations of a dynamical system, or in carry- 
ing out an approximation, we are accustomed to deal with numbers whose squares (and higher powers) are ignored : and $e$ will here be regarded as such a number. We may say with Prof. Study that $e^{2}=0$; or we may treat $e$ as a number whose square is neglected; or again we may assert that the statements we shall make are correct only to the first power of $v$. Whichever plan is adopted we have the fundamental relation connecting $L, M, N$.

$$
L^{2}+M^{2}+N^{2}=1
$$

With this convention as to $e,(L, M, N)$ are defined as the Dual Coordinates of the straight line.

Quantities such as $n+e r, u+e x$. . where $n, r, u, x$ . . . are ordinary numbers will be termed Dual Numbers. Powers and roots of a dual number, and the sum and product and quotient of two dual numbers ${ }^{1}$ are themselves dual numbers; an algebra of dual numbers may in fact be developed. If any three dual numbers, $u+e x, v+e y, w+e z$, are chosen there is just one line whose dual coordinates $L, M, N$ are proportional to them: to obtain this line, divide each of the three numbers by the square root of the sum of their squares. The ratios of any three dual numbers,

$$
\begin{aligned}
& U=u+e x \\
& V=v+e y \\
& W=v+e z,
\end{aligned}
$$

determine a certain straight line, and may be considered as " homogeneous dual coordinates" of the line; it will be seen that $u, v, w$ are the direction-ratios of the line. But $(L, M, N)$, defined above and satisfying (i), depend upon the directioncosines and so determine not only the line but a positive sense or direction upon it.

2. Had $L, M, N$ been ordinary numbers satisfying (i), geometry would interpret them as coordinates of a point on a sphere of unit radius. A number of results in spherical

1 Except sometimes if the number $u+e x$ has $u$ zero. 
geometry can, as Prof. Study shows, be interpreted in terms of dual numbers to give theorems upon lines in space. For example the condition

$$
L_{1} L_{2}+H_{1} M_{2}+N_{1} N_{2}=0,
$$

which expresses the fact that two points $\left(L_{1}, M_{1}, N_{1}\right)\left(L_{2}, M_{2}, N_{2}\right)$ are a quadrant apart, gives when interpreted in terms of the dual coordinates of two lines the condition that the two lines meet at right angles. Since the condition is homogeneous in each set of coordinates any factor may be rejected from each set and the condition holds equally in terms of the coordinates $U, V, W$, viz.,

$$
U_{1} U_{2}+V_{1} r_{2}+W_{1} W_{2}=0 .
$$

This is easily verified if $L, M, N$ are expressed in terms of $l, m, n, p, q, r$. Again the expression

$$
L_{1} L_{2}+M_{1} M_{2}+N_{1} N_{2}
$$

in general represents in spherical geometry the cosine of the angle subtended at the centre of the sphere by the two points. In dual coordinates, if we substitute for $L, M, N$ in terms of $l, m, n, p, q, r$, and drop terms in $\epsilon^{2}$, we obtain

$l_{1} l_{2}+m_{1} m_{2}+n_{1} n_{2}+e\left(l_{1} p_{2}+l_{2} p_{1}+m_{1} q_{2}+m_{2} q_{1}+n_{1} r_{2}+n_{2} r_{1}\right)$.

If $\theta$ is the angle between the positive directions of two lines in space, and $D$ is the length of their shortest distance (with a convention as to its sign), this formula represents

$$
\cos \theta-e D \sin \theta=\cos (\theta+e D)
$$

to the first power of $e$, Prof. Study calls $\theta+e D$ the dual anglc between the two lines. The vertices of a spherical triangle $A B C$ may be any three points of a sphere; the poles $A^{\prime}, B^{\prime}, C^{\prime}$ of the sides $B C, C A, A B$ are points such that $A^{\prime} B, A^{\prime} C$, etc., are quadrants. Extending this by the use of dual coordinates, so that $A, B, C$ represent three arbitrary lines in space, and $A^{\prime}, B^{\prime}, C^{\prime}$ represent the shortest distances of each two, he derives from the common formulae of spherical trigonometry relations connecting the dual angles (i.e., connecting both the angles and the shortest distances) between these lines. See Geometrie der Dynamen, pp. 209-213. 
3. The object of this paper is to obtain in a precisely similar manner certain properties of Ruled Surfaces : the first and most fundamental of these properties is noted by Study. They are derived from properties of curves on a sphere; the coordinates of points are replaced by dual numbers and are interpreted by means of lines in space.

With any spherical curve is associated a second curve, the polar curve of the first. If $X, Y, Z$ are points of a given curve and lengths $X X^{\prime}, Y Y^{\prime}, Z Z^{\prime}$, each equal to a quadrant are measured along the normals at $X, Y, Z$, then $X^{\prime}, Y^{\prime} Z^{\prime}$ are the corresponding points of the polar curve. Each of the curves is the polar of the other, and the arcs $X X^{\prime}$ $Y Y^{\prime}, Z Z^{\prime}$ are normal to both. If the great circles which touch the two curves at $X$ and $X^{\prime}$ meet in $T$, and those which touch them at $Y$ and $Y^{\prime}$ meet in $U, T$ and $U$ are the poles of the arcs $X X^{\prime}$ and $Y Y^{\prime}$ respectively, and the two spherical triangles $X X^{\prime} T$ and $Y Y^{\prime} U$ have all their sides quadrants. Further, if the arcs $X X^{\prime}$ and $Y Y^{\prime}$ meet in $C, C$ is the pole of $T U$; and as $X$ and $Y$ approach coincidence the curve which is the locus of the ultimate position of $C$ is polar to the curve which is the locus of $T$ and $U$. The ultimate position of $C$ is the common centre of curvature of the given curve and the polar curve, and its locus is their evolute.

Next imagine a Ruled Surface formed by a simple infinity of generating lines; each has dual coordinates $L, M, N$. We have a simple infinity of values of $L, M, N$, all satisfying (i), and the fact that they are dual numbers does not prevent us from deriving a second set of values in the same way that the coordinates of $X^{\prime}, Y^{\prime} Z^{\prime}$ were derived from those of $X$, $Y, Z$. They represent the generators of a second ruled surface; and, since $X X^{\prime}, Y Y^{\prime} Z Z^{\prime}$ are quadrants, corresponding generators of the two surfaces intersect at right angles. But, further, since $X^{l}$ is the pole of the great circle tangent at $X$, and $X$ the pole of that at $X^{\prime}$, the generator of each ruled surface is the shortest distance of two generators of the other, when they move up to coincidence. The plane containing corresponding generators of the two ruled surfaces 
touches both the surfaces. In fact not only do corresponding generators of the given and the derived ruled surfaces intersect at right angles, but the locus of their point of intersection is the curve of striction on both surfaces, and the two surfaces touch at each point of the curve. This is the surface noticed by Study and called by him the Strictionsband; there is however something to be said for adopting here and elsewhere as far as possible the names used in spherical geometry with the word dual prefixed; we may term the second ruled surface the dual polar of the first.

To the points such as $T$, the pole of the common normal $X X^{\prime}$ of the given spherical curve and its polar, correspond lines which intersect both the two corresponding generators of the given ruled surface and its dual polar surface perpendicularly, i.e., normals to the two ruled surfaces at points of their line of striction. These normals form a third ruled surface-the "surface of striction-normals" will serve to describe it-corresponding to the locus of points such as $T$ and $U$ of the spherical curves.

When three straight lines are taken in space and a positive direction is assigned to each, there is a unique line which is equally inclined to the three and is equidistant from the three. If the three lines are chosen from among the generators of the given ruled surface and approach coincidence, the line whose inclination and shortest distance from each is the same takes a definite limiting position. Spherical geometry enables us to determine it. The line in question, whose dual distances from three neighbouring generators are equal, corresponds to a point on the sphere whose distances from three points of the curve are equal; the limiting position of this point is $C$, the pole of $T U$ and the centre of curvature of the curve at $X$. We infer that if we take the normals to the given ruled surface at two neighbouring points of its line of striction and construct their common perpendicular or shortest distance, the ultimate position of this line when the two neighbouring points move up to coincidence is the required line of equal inclination and equal distance from three generators. 
4. It is clear from the geometry of the sphere that the same line is also the line of equal inclination and distance from three-generators of the Strictionsband or dual polar surface. But it is also clear that the Strictionsband is merely one of an infinity of surfaces having similar properties. For, on the sphere, an infinity of curves (as well as the polar curve) have the same evolute as a given curve: we may measure any constant angular distance along the normals of the given curve and we shall obtain such a curve. Hence in space, given a ruled surface and its curve of striction, if we rotate each generator through a right angle round the normal at the point where the generator meets the curve of striction, we arrive at the Strictionsband of Prof. Study, which has the same curve of striction and touches the given surface at each point of that curve: but if we had rotated each generator about the same normal through any constant angle, we should have obtained a different ruled surface having the same properties. There are in fact an infinity of ruled surfaces having a common curve of striction and having the same tangent plane at each point of that curve. Further for the constant angle of rotation we may substitute a constant dual angle; in place of a simple rotation about the normal we may impose upon each generator a rotation through a constant angle about the normal plus a translation through a constant distance along that normal. We arrive at a new ruled surface having-not of course the same curve of striction-but the same surface of striction-normals as the given surface. In this doubly infinite system of ruled surfaces the original surface is not distinct from the rest. The foundation of the figure is the surface of striction-normals. The generators of the other surfaces form the totality of lines which meet the striction-normals perpendicularly, and they are grouped into surfaces in a manner suggested by the system of curves on a sphere.

But on the sphere the curves are derived more simply from the locus of $C$ than from that of $T$; the former is the evolute and is the polar of the latter. It may be imagined that an inextensible thread lying on the surface of the sphere and kept always taut is unwrapped from the evolute; or that, 
if the evolute is replaced by a polygon formed of a number of small arcs of great circles, and $A B, B C, C D, D E$ are the sides of polygon starting from a point $A$ of the evolute, first $A B$ is turned about $B$ until it becomes the continuation of $C B$, then $A B$ and $B C$ together are turned about $C$ until they become the continuation of $D C$, and so on. The curve described by $A$ ultimately becomes an involute, one of the curves desired. The other involutes, described by other points than $A$, can be obtained by lengthening or shortening the arc of the great circle which is normal to the locus of $A$ by a constant amount. It is possible to imagine a somewhat similar method of obtaining the ruled surfaces.

5. In place of the surface of striction-normals (which corresponds to the locus of $T$ ), we prefer to use its dual polar surface (which corresponds to the evolute, or locus of $C$ ) and which we describe as the dual evolute; we have seen that it is the locus of lines of equal inclination and distance from three generators of any of the system of ruled surfaces, when the generators approach coincidence. In discussing the sphere we took a series of points on the evolute and so substituted a polygon for the continuous curve; here we will select a series of generators of the dual evolute at small distances apart and disregard the intermediate generators. The developing or unwrapping of the locus on the sphere transformed it step by step into a great circle without altering the length of its elements. Now to points on a great circle correspond in space (when dual numbers are introduced as coordinates) lines which meet a certain line at right angles, and a surface formed by such lines is known as a right conoid. We have to imagine a method by which the serics of generators of the dual evolute are transformed into generators of a right conoid without alteration of the dual angle (i.e., of the angle and shortest distance) between each successive two. This is not difficult. Call the successive generators $a, b, c, d, c$. . and the shortest distances $(a b),(b c),(c d)$. . . Imagine $a$ and $(a b)$ translated parallel to $b$ until the ends of $(a b)$ and $(b c)$ which lie on $b$ 
coincide; then imagine $a$ and $(a b)$ rotated about $b$ until (ab) comes into line with $(b c): a, b,(a b),(b c)$ may now be supposed to be fixed together and to move together like a rigid body. Translate them parallel to $c$ until the point where (bc) meets $c$ is brought into coincidence with the point where $(c d)$ meets $c$ and rotate them about $c$ until the line made up of $(a b)$ and $(b c)$ comes into line with $(c d): a, b, c(a b)$, $(b c),(c d)$ may now be supposed fixed together, $(a b),(b c)$, $(c d)$ being in a straight line and $a, b, c$ meeting at right angles. In the same way a translation parallel to $d$ and a rotation about $d$ enables us to combine another generator $d$ and another shortest distance $(d e)$. This gives an idea how the line $a$ traces out one of the ruled surfaces; lines rigidly connected with $a$ and $(a b)$, meeting $(a b)$ at a fixed distance from $a$ and inclined to $a$ at a fixed angle, trace out other ruled surfaces of the system.

The methods here used will obviously lead to other results, if it is thought desirable to pursue them. It will however be recognised that the properties of the curve of striction which have been given can be obtained without putting pen to paper, when once the principle of Professor Study's dual coordinates is understood. 